\title{
Synthesis and preliminary screening of benzothiazol-2-yl thiadiazole derivatives for anticonvulsant activity
}

\author{
NADEEM SIDDIQUI ${ }^{1 *}$ \\ ARPANA RANA ${ }^{1}$ \\ SUROOR A. KHAN ${ }^{1}$ \\ S. EHTAISHAMUL HAQUE ${ }^{2}$ \\ M. FAIZ ARSHAD ${ }^{1}$ \\ SHARIQUE AHMED 3 \\ WAQUAR AHSAN ${ }^{1}$ \\ ${ }^{1}$ Department of Pharmaceutical \\ Chemistry, Faculty of Pharmacy \\ Hamdard University \\ New Delhi-110062, India \\ ${ }^{2}$ Department of Pharmacology, Faculty \\ of Pharmacy, Hamdard University \\ New Delhi-110062, India \\ ${ }^{3}$ Department of Biochemistry, Faculty \\ of Medicine, $7^{\text {th }}$ October University \\ Misurata, Libya
}

\begin{abstract}
Various $N$-(5-chloro-6-substituted-benzothiazol-2-yl)- $N$ '-(substituted phenyl)-[1,3,4]thiadiazole-2,5-diamines (5a-t) were designed and synthesized starting from substituted acetophenones. Structures of all the compounds were confirmed on the basis of spectral and elemental analyses. All the newly synthesized compounds were screened for their anticonvulsant activity and were compared with the standard drug phenytoin sodium. Interestingly, all the compounds showed protections against seizures in the range $50-100 \%$ indicative of the promising nature of the compounds against seizure spread. Compounds $\mathbf{5 b}$ and $5 \mathrm{c}$ showed complete protection against MES induced seizures.

Keywords: benzothiazole, thiadiazole, anticonvulsant, neurotoxicity, hepatotoxicity
\end{abstract}

Accepted July 27, 2009

The term epilepsy is a collective term that includes disorders of the brain function characterized by the periodic and unpredictable occurrence of seizures. Epilepsies are common and frequently devastating and affect around 1-2\% of the world population (1).

Current drug therapy for epilepsy suffers from a number of disadvantages, including the fact that the convulsions of approximately $25 \%$ of epileptics are inadequately controlled by medication (2). In recent years, the field of antiepileptic drug development has become quite dynamic, affording many promising research opportunities.

In our previous study (3-5), we designed aryl-substituted semicarbazones with benzothiazole moiety, in which we made some modifications in the structure of semicarbazones. The lipophilic aryl ring was replaced with a versatile heterocyclic molecule ben-

*Correspondence; e-mail: nadeems_03@yahoo.co.in, nadeems_03@rediffmail.com 
N. Siddiqui et al.: Synthesis and preliminary screening of benzothiazol-2-yl thiadiazole derivatives for anticonvulsant activity, Acta Pharm. 59 (2009) 441-451.

zothiazole, which possesses significant anticonvulsant properties. On the other hand, thiadiazole moiety was also found to possess significant anticonvulsant activity $(6,7)$. In continuation of our previous research on benzothiazoles, we report here the synthesis and anticonvulsant activity of several $N$-(5-chloro-6-substituted-benzothiazol-2-yl)- $N$ '-(substituted phenyl)-[1,3,4]thiadiazole-2,5-diamines. Incorporation of 1,3,4-thiadiazole moiety into the second position of the benzothiazole ring may result in compounds having better anticonvulsant activity. The neurotoxicity as well as liver toxicity of the compounds has also been evaluated.

\section{EXPERIMENTAL}

The chemicals and solvents used for the experimental work were commercially procured from E. Merck, CDH, S. D. Fine Chem. and Qualigens, all from India. The silica gel G used for analytical chromatography (TLC) was obtained from E. Merck, India. Melting points were determined in an open glass capillary using a Kjeldahl flask containing paraffin and are uncorrected. The proton magnetic resonance spectra ( $\left.{ }^{1} \mathrm{H} \mathrm{NMR}\right)$ were recorded on a Bruker $300 \mathrm{MHz}$ instrument (Bruker, Germany) in DMSO- $d_{6}$ using tetramethylsilane $\left[\left(\mathrm{CH}_{3}\right)_{4} \mathrm{Si}\right]$ as internal standard. Chemical shifts $(\delta)$ are expressed in ppm. The infrared spectra of compounds (in $\mathrm{cm}^{-1}$ ) were recorded in $\mathrm{KBr}$ on a Bio-Rad FTIR (Browser Morner, USA) spectrometer. Elemental analyses were performed on a 240c analyzer (Perkin Elmer, USA).

\section{Syntheses}

5-Chloro-6-substituted-benzothiazol-2-ylamines (1a,b). - A mixture of substituted aniline $(0.01 \mathrm{~mol})$ and potassium thiocyanate $(0.01 \mathrm{~mol})$ in glacial acetic acid was cooled and stirred. To this solution, bromine $(0.01 \mathrm{~mol})$ was added dropwise at such a rate to keep the reaction temperature below $10{ }^{\circ} \mathrm{C}$ throughout the addition. Stirring was continued for additional $3 \mathrm{~h}$ and the separated hydrochloride salt was filtered, washed with acetic acid and dried. It was dissolved in hot water and neutralized with aqueous ammonia solution ( $25 \%$ ). The precipitate obtained was filtered, washed with water, dried and recrystallized to afford the 6-substituted-1,3-benzothiazol-2-amine (1a,b). Yield: 64 \%; m.p. $248{ }^{\circ} \mathrm{C}$; IR (KBr) cm -1 : $3239\left(\mathrm{NH}_{2}\right), 3016(\mathrm{CH}-\mathrm{Ar}), 1562(\mathrm{C}=\mathrm{N}), 804(\mathrm{C}-\mathrm{Cl}) ;{ }^{1} \mathrm{H}$ NMR (DMSO- $\left.d_{6}\right) \delta$ ppm: 7.89-8.16 (m, 2H, ArH), $9.76\left(\mathrm{~s}, 2 \mathrm{H}, \mathrm{NH}_{2}, \mathrm{D}_{2} \mathrm{O}\right.$ exchangeable).

(5-Chloro-6-substituted-benzothiazol-2-yl)-ureas $(\mathbf{2} \mathbf{a}, \mathbf{b})$. - To a solution of sodium cyanate $(0.5 \mathrm{~g})$ in minimum quantity of water, glacial acetic acid $(5 \mathrm{~mL})$ was added. This solution was heated with the respective 2-amino-6-substituted benzothiazoles ( $1.7 \mathrm{~g}, 0.01$ $\mathrm{mol}$ ) in alcohol till the mixture contents became turbid and the volume was half of the original volume. The contents were added to ice cool water. The solid obtained was filtered off and dried. Yield: 73 \%; m.p. $262^{\circ} \mathrm{C}$; IR (KBr) cm ${ }^{-1}: 3218(\mathrm{NH}), 3165\left(\mathrm{NH}_{2}\right), 2937$ (CH-Ar), $1678(\mathrm{C}=\mathrm{O}), 1545(\mathrm{C}=\mathrm{N}), 816(\mathrm{C}-\mathrm{Cl}) ;{ }^{1} \mathrm{H}$ NMR (DMSO-d 6 ) $\delta$ ppm: 7.82-8.20 (m, $2 \mathrm{H}, \mathrm{ArH}), 9.14$ (s, 2H, $\mathrm{NH}_{2}, \mathrm{D}_{2} \mathrm{O}$ exchangeable), 9.86 (s, 1H, NH, $\mathrm{D}_{2} \mathrm{O}$ exchangeable).

N-(5-Chloro-6-substituted-benzothiazol-2-yl)hydrazine carboxamides (3a,b). - To a solution of substituted benzothiazole urea $(2 \mathbf{a}, \mathbf{b}, 0.01 \mathrm{~mol})$ in ethanol, hydrazine hydrate $(0.01$ 
$\mathrm{mol})$ and $\mathrm{NaOH}(0.04 \mathrm{~g})$ were added. The resulting mixture was refluxed for $16 \mathrm{~h}$. It was then concentrated, cooled and poured over crushed ice to get the precipitate, which was filtered, washed with water and recrystallized from ethanol to get hydrazine carboxamides. Yield: $71 \%$; m. p. $224^{\circ} \mathrm{C}$; IR (KBr) cm${ }^{-1}$ : 3265, $3218(\mathrm{NH}), 3112\left(\mathrm{NH}_{2}\right), 3017$ (CH-Ar), $1683(\mathrm{C}=\mathrm{O}), 1513(\mathrm{C}=\mathrm{N}), 845(\mathrm{C}-\mathrm{Cl}) ;{ }^{1} \mathrm{H}$ NMR $\left(\mathrm{DMSO}-d_{6}\right) \delta \mathrm{ppm:}$ 7.74-8.12 (m, 2H, ArH), $8.98\left(\mathrm{~s}, 2 \mathrm{H}, \mathrm{NH}_{2}, \mathrm{D}_{2} \mathrm{O}\right.$ exchangeable), $9.41\left(\mathrm{~s}, 1 \mathrm{H}, \mathrm{NH}, \mathrm{D}_{2} \mathrm{O}\right.$ exchangeable), $9.86(\mathrm{~s}, 1 \mathrm{H}$, $\mathrm{NH}, \mathrm{D}_{2} \mathrm{O}$ exchangeable).

N-(5-Chloro-6-substituted-benzothiazol-2-yl)-2-[(4-substituted phenyl)carbamothioyl] hydrazinecarboxamides (4a-t). - A solution of carboxamides in glacial acetic acid (5 mL) and ethanol $(10 \mathrm{~mL})$ was heated to boiling and refluxed with aromatic ketones $(1 \mathrm{~g}, 0.122 \mathrm{~mol})$ for $5 \mathrm{~h}$. Refluxed solution was cooled to room temperature and kept overnight. The solid was collected out, washed with methanol, dried and recrystallized from ethanol to get the target compound. Yield: 58 \%; m.p. $269{ }^{\circ} \mathrm{C}$; IR (KBr) cm $\mathrm{cm}^{-1}$ : 3285, 3245, 3212, 3176 (NH), 3006 (CH-Ar), 1673 (C=O), 1534 (C=N), 1055 (C=S), 832 (C-Cl); ' ${ }^{1} \mathrm{H}$ NMR (DMSO$\left.-d_{6}\right) \delta$ ppm: 6.76-8.32 (m, 7H, ArH), $8.52\left(\mathrm{~s}, 1 \mathrm{H}, \mathrm{NH}, \mathrm{D}_{2} \mathrm{O}\right.$ exchangeable), $8.75(\mathrm{~s}, 1 \mathrm{H}$, $\mathrm{NH}, \mathrm{D}_{2} \mathrm{O}$ exchangeable), $9.18\left(\mathrm{~s}, 1 \mathrm{H}, \mathrm{NH}, \mathrm{D}_{2} \mathrm{O}\right.$ exchangeable), $9.49\left(\mathrm{~s}, 1 \mathrm{H}, \mathrm{NH}, \mathrm{D}_{2} \mathrm{O}\right.$ exchangeable).

$\mathrm{N}-(5-C h l o r o-6-s u b s t i t u t e d-b e n z o t h i a z o l-2-y l)-\mathrm{N}^{\prime}-($ substituted phenyl)-[1,3,4]thiadiazole-2,5-diamines (5a-t). - To cold concentrated sulphuric acid ( $25 \mathrm{~mL})$, compounds (4a-t, 0.01 mol) were added gradually under constant stirring. After the addition was completed, the solution was kept at room temperature for $2 \mathrm{~h}$ and poured onto crushed ice. The solid thus separated was washed with water and treated with a dilute solution of sodium bicarbonate. The product obtained was washed with water, dried and recrystallized from ethanol to afford the titled compounds (5a-t).

\section{Pharmacology}

Anticonvulsant activity. - Anticonvulsant evaluation was undertaken using a reported procedure $(8,9)$. Albino mice (Swiss, 25-30 g) were used in groups of six each as experimental animals. The test compounds and standard drug were suspended in Tween $80(1 \%)$ or in a $0.5 \%$ methyl cellulose-water mixture and administered intraperitoneally. The animals were maintained on an adequate diet and allowed free access to food and water except during the short time they were removed from cages for testing. The animals were maintained at room temperature $\left(25 \pm 2{ }^{\circ} \mathrm{C}\right)$. All the experimental protocols were carried out with the permission of the Institutional Animal Ethics Committee (IAEC). Animals were obtained from the Central Animal House Facility, Jamia Hamdard University, New Delhi, India.

Maximal electroshock seizure test (MES). - Maximal electroshock seizure was elicited with a 60 cycle alternating current of $50 \mathrm{~mA}$ intensity delivered for $0.25 \mathrm{~s}$ via ear clip electrodes. Maximal seizure typically consists of a short period of tonic extension of the hind limbs and a final clonic episode. Abolition of the hind limb tonic extensor component of the seizure is defined as protection and the results are expressed as $\%$ protection. 
Neurotoxicity (NT). - The rotarod test was used to evaluate neurotoxicity (10). The animal was placed on a 3.2-cm diameter knurled rod rotating at $6 \mathrm{rpm}$. Normal mice can remain indefinitely on a rod rotating at this speed. Neurological toxicity is defined as the failure of the animal to remain on the rod for $1 \mathrm{~min}$. Results are expressed as the number of animals exhibiting toxicity/number of animals tested.

Assessment of liver function. - Biochemical parameters such as serum glutamate oxaloacetate transaminase (SGOT) and serum glutamate pyruvate transaminase (SGPT) were estimated by the Reitman and Frankel method (11) and alkaline phosphatase was measured using the King and Armstrong method (12). Total protein and total albumin were also measured according to the biuret method (13).

Statistical analyses. - All the statistical analyses were performed using the SigmaStat version 4.0 software and ANOVA followed by Dunnett's multiple comparison test.

\section{RESULTS AND DISCUSSION}

$N$-(5-chloro-6-substituted-benzothiazol-2-yl)-N'-(substituted phenyl)-[1,3,4]thiadiazole-2,5-diamines (5a-t) were synthesized by the sequence described in Scheme 1 . Initial compounds, 5-chloro-6-substituted-1,3-benzothiazol-2-amines (1a,b), were synthesized by treating arylamines with potassium thiocyanate. The 5-chloro-6-substituted-benzothiazol-2-yl-ureas $(\mathbf{2} \mathbf{a}, \mathbf{b})$ were prepared by treating appropriate benzothiazole with sodium cyanate in the presence of glacial acetic acid. Further $N$-(5-chloro-6-substituted-1,3-benzothiazol-2-yl) hydrazine carboxamides $(\mathbf{3} \mathbf{a}, \mathbf{b})$ were obtained by refluxing them with<smiles>[R]c1cc2sc(NC(N)=O)nc2cc1Cl</smiles><smiles>[R]c1ccc(NC(=S)NNC(=O)Nc2nc3cc(Cl)c([R])cc3s2)cc1</smiles>

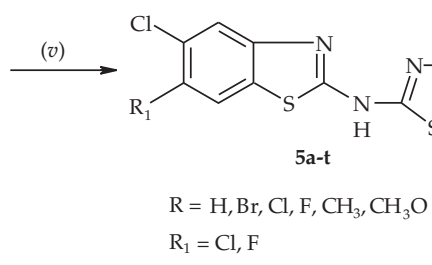

Reagents and conditions: (i) glacial acetic acid, $\mathrm{Br}_{2}$ (ii) glacial acetic acid, $\mathrm{NaOCN}$, (iii) conc. $\mathrm{NaOH} / \mathrm{EtOH}, \mathrm{NH}_{2} \mathrm{NH}_{2} \cdot \mathrm{H}_{2} \mathrm{O}$, (iv) ArNCS, abs. ethanol, reflux, (v) $\mathrm{H}_{2} \mathrm{SO}_{4}$

Scheme 1 
hydrazine hydrate to yield carboxamides. The final compounds (5a-t) were obtained by refluxing carboxamides with different substituted phenyl isothiocyanates in ethanol and then cyclizing them in the presence of cold concentrated sulphuric acid. Physicochemical parameters of the synthesized compounds are presented in Table I.

The structures and purity of the final compounds were confirmed on the basis of spectral and elemental analyses and the data were within $\pm 0.4 \%$ of theoretical values

Table I. Physicochemical data of synthesized compounds 5a-t

\begin{tabular}{|c|c|c|c|c|c|c|c|c|}
\hline \multirow{2}{*}{$\begin{array}{l}\text { Compd. } \\
\text { No. }\end{array}$} & \multirow{2}{*}{$\mathrm{R}$} & \multirow{2}{*}{$\mathrm{R}_{1}$} & \multirow{2}{*}{$\begin{array}{l}\text { Mol. formula } \\
M_{\mathrm{r}}{ }^{\mathrm{a}}\end{array}$} & \multicolumn{3}{|c|}{ Found/calcd. (\%) } & \multirow{2}{*}{$\begin{array}{c}\text { Yield } \\
(\%)\end{array}$} & \multirow{2}{*}{$\begin{array}{l}\text { M.p. } \\
\left({ }^{\circ} \mathrm{C}\right)^{b}\end{array}$} \\
\hline & & & & C & $\mathrm{H}$ & $\mathrm{N}$ & & \\
\hline $5 a$ & $4-\mathrm{H}$ & $\mathrm{Cl}$ & $\begin{array}{c}\mathrm{C}_{15} \mathrm{H}_{9} \mathrm{Cl}_{2} \mathrm{~N}_{5} \mathrm{~S}_{2} \\
(394.29)\end{array}$ & $\begin{array}{l}45.65 \\
45.69\end{array}$ & $\begin{array}{l}2.32 \\
2.30\end{array}$ & $\begin{array}{l}17.77 \\
17.76\end{array}$ & 55 & $259-261$ \\
\hline $5 b$ & $4-\mathrm{Br}$ & $\mathrm{Cl}$ & $\begin{array}{c}\mathrm{C}_{15} \mathrm{H}_{8} \mathrm{BrCl}_{2} \mathrm{~N}_{5} \mathrm{~S}_{2} \\
(473.19)\end{array}$ & $\begin{array}{l}38.12 \\
38.07\end{array}$ & $\begin{array}{l}1.67 \\
1.70\end{array}$ & $\begin{array}{l}14.78 \\
14.80\end{array}$ & 45 & $279-281$ \\
\hline $5 c$ & $3-\mathrm{Cl}$ & $\mathrm{Cl}$ & $\begin{array}{c}\mathrm{C}_{15} \mathrm{H}_{8} \mathrm{Cl}_{3} \mathrm{~N}_{5} \mathrm{~S}_{2} \\
(428.74)\end{array}$ & $\begin{array}{l}42.03 \\
42.02\end{array}$ & $\begin{array}{l}1.82 \\
1.88\end{array}$ & $\begin{array}{l}16.37 \\
16.33\end{array}$ & 50 & $257-159$ \\
\hline $5 d$ & $4-\mathrm{Cl}$ & $\mathrm{Cl}$ & $\begin{array}{c}\mathrm{C}_{15} \mathrm{H}_{8} \mathrm{Cl}_{3} \mathrm{~N}_{5} \mathrm{~S}_{2} \\
(428.74)\end{array}$ & $\begin{array}{l}42.00 \\
42.02\end{array}$ & $\begin{array}{l}1.84 \\
1.88\end{array}$ & $\begin{array}{l}16.37 \\
16.33\end{array}$ & 54 & $261-263$ \\
\hline $5 e$ & $4-\mathrm{F}$ & $\mathrm{Cl}$ & $\begin{array}{c}\mathrm{C}_{15} \mathrm{H}_{8} \mathrm{Cl}_{2} \mathrm{FN}_{5} \mathrm{~S}_{2} \\
(412.28)\end{array}$ & $\begin{array}{l}43.72 \\
43.70\end{array}$ & $\begin{array}{l}1.97 \\
1.96\end{array}$ & $\begin{array}{l}16.96 \\
16.99\end{array}$ & 60 & $265-267$ \\
\hline $5 f$ & $3-\mathrm{CH}_{3}$ & $\mathrm{Cl}$ & $\begin{array}{c}\mathrm{C}_{16} \mathrm{H}_{11} \mathrm{Cl}_{2} \mathrm{~N}_{5} \mathrm{~S}_{2} \\
(408.32)\end{array}$ & $\begin{array}{l}47.04 \\
47.06\end{array}$ & $\begin{array}{l}2.74 \\
2.72\end{array}$ & $\begin{array}{l}17.12 \\
17.15\end{array}$ & 50 & $267-269$ \\
\hline $5 \mathrm{~g}$ & $4-\mathrm{CH}_{3}$ & $\mathrm{Cl}$ & $\begin{array}{c}\mathrm{C}_{16} \mathrm{H}_{11} \mathrm{Cl}_{2} \mathrm{~N}_{5} \mathrm{~S}_{2} \\
(408.32)\end{array}$ & $\begin{array}{l}46.03 \\
46.06\end{array}$ & $\begin{array}{l}2.75 \\
2.72\end{array}$ & $\begin{array}{l}17.19 \\
17.15\end{array}$ & 54 & $249-251$ \\
\hline $5 \mathrm{~h}$ & $2-\mathrm{OCH}_{3}$ & $\mathrm{Cl}$ & $\begin{array}{c}\mathrm{C}_{16} \mathrm{H}_{11} \mathrm{Cl}_{2} \mathrm{~N}_{5} \mathrm{OS}_{2} \\
(424.32)\end{array}$ & $\begin{array}{l}45.26 \\
45.29\end{array}$ & $\begin{array}{l}2.59 \\
2.61\end{array}$ & $\begin{array}{l}15.53 \\
16.50\end{array}$ & 48 & 239-241 \\
\hline $5 i$ & $3-\mathrm{OCH}_{3}$ & $\mathrm{Cl}$ & $\begin{array}{c}\mathrm{C}_{16} \mathrm{H}_{11} \mathrm{Cl}_{2} \mathrm{~N}_{5} \mathrm{OS}_{2} \\
(424.32)\end{array}$ & $\begin{array}{l}47.26 \\
47.29\end{array}$ & $\begin{array}{l}2.64 \\
2.61\end{array}$ & $\begin{array}{l}16.51 \\
16.50\end{array}$ & 45 & $243-245$ \\
\hline $5 j$ & $4-\mathrm{OCH}_{3}$ & $\mathrm{Cl}$ & $\begin{array}{c}\mathrm{C}_{16} \mathrm{H}_{11} \mathrm{Cl}_{2} \mathrm{~N}_{5} \mathrm{OS}_{2} \\
(424.32)\end{array}$ & $\begin{array}{l}45.31 \\
45.29\end{array}$ & $\begin{array}{l}2.64 \\
2.61\end{array}$ & $\begin{array}{l}16.54 \\
16.50\end{array}$ & 45 & $269-271$ \\
\hline $5 \mathrm{k}$ & $4-\mathrm{H}$ & $\mathrm{F}$ & $\begin{array}{c}\mathrm{C}_{15} \mathrm{H}_{9} \mathrm{ClFN}_{5} \mathrm{~S}_{2} \\
(377.84)\end{array}$ & $\begin{array}{l}47.71 \\
47.68\end{array}$ & $\begin{array}{l}2.42 \\
2.40\end{array}$ & $\begin{array}{l}18.58 \\
18.54\end{array}$ & 65 & $255-257$ \\
\hline 51 & $4-\mathrm{Br}$ & $\mathrm{F}$ & $\begin{array}{c}\mathrm{C}_{15} \mathrm{H}_{8} \mathrm{BrClFN}_{5} \mathrm{~S}_{2} \\
(456.73)\end{array}$ & $\begin{array}{l}39.41 \\
39.45\end{array}$ & $\begin{array}{l}1.81 \\
1.77\end{array}$ & $\begin{array}{l}15.30 \\
15.33\end{array}$ & 50 & $253-255$ \\
\hline $5 \mathrm{~m}$ & $3-\mathrm{Cl}$ & $\mathrm{F}$ & $\begin{array}{c}\mathrm{C}_{15} \mathrm{H}_{8} \mathrm{Cl}_{2} \mathrm{FN}_{5} \mathrm{~S}_{2} \\
(412.28)\end{array}$ & $\begin{array}{l}43.73 \\
43.70\end{array}$ & $\begin{array}{l}1.92 \\
1.96\end{array}$ & $\begin{array}{l}16.96 \\
16.99\end{array}$ & 64 & $247-249$ \\
\hline $5 n$ & $4-\mathrm{Cl}$ & $\mathrm{F}$ & $\begin{array}{c}\mathrm{C}_{15} \mathrm{H}_{8} \mathrm{Cl}_{2} \mathrm{FN}_{5} \mathrm{~S}_{2} \\
(412.28)\end{array}$ & $\begin{array}{l}43.67 \\
43.70\end{array}$ & $\begin{array}{l}1.99 \\
1.96\end{array}$ & $\begin{array}{l}16.96 \\
16.99\end{array}$ & 64 & $229-231$ \\
\hline
\end{tabular}


N. Siddiqui et al.: Synthesis and preliminary screening of benzothiazol-2-yl thiadiazole derivatives for anticonvulsant activity, Acta Pharm. 59 (2009) 441-451.

\begin{tabular}{|c|c|c|c|c|c|c|c|c|}
\hline 50 & $4-\mathrm{F}$ & $\mathrm{F}$ & $\begin{array}{c}\mathrm{C}_{15} \mathrm{H}_{8} \mathrm{ClF}_{2} \mathrm{~N}_{5} \mathrm{~S}_{2} \\
(395.83)\end{array}$ & $\begin{array}{l}45.50 \\
45.52\end{array}$ & $\begin{array}{l}1.99 \\
2.04\end{array}$ & $\begin{array}{l}17.67 \\
17.69\end{array}$ & 60 & $227-229$ \\
\hline $5 p$ & $3-\mathrm{CH}_{3}$ & $\mathrm{~F}$ & $\begin{array}{c}\mathrm{C}_{16} \mathrm{H}_{11} \mathrm{ClFN}_{5} \mathrm{~S}_{2} \\
(391.86)\end{array}$ & $\begin{array}{l}49.00 \\
49.04\end{array}$ & $\begin{array}{l}2.86 \\
2.83\end{array}$ & $\begin{array}{l}17.90 \\
17.87\end{array}$ & 66 & $225-227$ \\
\hline $5 q$ & $4-\mathrm{CH}_{3}$ & $\mathrm{~F}$ & $\begin{array}{c}\mathrm{C}_{16} \mathrm{H}_{11} \mathrm{ClFN}_{5} \mathrm{~S}_{2} \\
(391.86)\end{array}$ & $\begin{array}{l}49.01 \\
49.04\end{array}$ & $\begin{array}{l}2.86 \\
2.83\end{array}$ & $\begin{array}{l}17.91 \\
17.87\end{array}$ & 62 & $219-221$ \\
\hline $5 r$ & $2-\mathrm{OCH}_{3}$ & $\mathrm{~F}$ & $\begin{array}{c}\mathrm{C}_{16} \mathrm{H}_{11} \mathrm{ClFN}_{5} \mathrm{OS}_{2} \\
(407.86)\end{array}$ & $\begin{array}{l}47.14 \\
47.12\end{array}$ & $\begin{array}{l}2.69 \\
2.72\end{array}$ & $\begin{array}{l}17.21 \\
17.17\end{array}$ & 52 & $221-223$ \\
\hline $5 s$ & $3-\mathrm{OCH}_{3}$ & $\mathrm{~F}$ & $\begin{array}{c}\mathrm{C}_{16} \mathrm{H}_{11} \mathrm{ClFN}_{5} \mathrm{OS}_{2} \\
(407.86)\end{array}$ & $\begin{array}{l}47.17 \\
47.12\end{array}$ & $\begin{array}{l}2.70 \\
2.72\end{array}$ & $\begin{array}{l}17.13 \\
17.17\end{array}$ & 50 & 235-237 \\
\hline $5 t$ & $4-\mathrm{OCH}_{3}$ & $\mathrm{~F}$ & $\begin{array}{c}\mathrm{C}_{16} \mathrm{H}_{11} \mathrm{ClFN}_{5} \mathrm{OS}_{2} \\
(407.86)\end{array}$ & $\begin{array}{l}46.15 \\
46.12\end{array}$ & $\begin{array}{l}2.70 \\
2.72\end{array}$ & $\begin{array}{l}17.21 \\
17.17\end{array}$ & 62 & $245-247$ \\
\hline
\end{tabular}

a Solvent of crystallization: methanol.

$\mathrm{b}$ Melting point of the compounds at decomposition.

(Table II). The FTIR spectra showed bands at 3370-3153 $\mathrm{cm}^{-1}$ for the NH and 1674-1633 and $720-689 \mathrm{~cm}^{-1}$ for $\mathrm{C}=\mathrm{O}$ and $\mathrm{C}-\mathrm{S}-\mathrm{C}$, respectively. The ${ }^{1} \mathrm{H}$ NMR spectra showed two singlets at $\delta 8.85-9.30$ and $11.14-11.78$ ppm for the two NH protons.

\section{Pharmacology}

1-(5,6-Disubstituted-1,3-benzothiazol-2-yl)-3-[5-(4-substituted phenyl)-1,3,4-thiadiazol-2-yl urea derivatives $(5 \mathbf{a}-\mathbf{t})$ obtained from the reactional sequence were injected intraperitoneally into mice and evaluated by the maximal electroshock (MES), neurotoxicity screen using rotarod at the dose of $30 \mathrm{mg} \mathrm{kg}^{-1}$ body mass and observations were carried out at two different time intervals of 0.5 and $4 \mathrm{~h}$. The data are presented in Table III. Phenytoin was used as the standard for the comparison at the dose level of $30 \mathrm{mg}$ $\mathrm{kg}^{-1}$. All the compounds showed anti-MES activity indicative of their ability to prevent seizure spread. The compounds that showed $100 \%$ protection against the MES model at $30 \mathrm{mg} \mathrm{kg}^{-1}$ body mass were $\mathbf{5 b}, \mathbf{5} \mathbf{c}$ both after 0.5 and $4 \mathrm{~h}$. Compounds $\mathbf{5 k}, \mathbf{5 o}, \mathbf{5 e}$ and $\mathbf{5 f}$ showed $83 \%$ protection at both time intervals except for compound 5o, which showed $66 \%$ protection after $4 \mathrm{~h}$, indicating a rapid onset and shorter duration of action. Compounds $5 \mathrm{~d}, \mathbf{5 j}, \mathbf{5 l}, \mathbf{5 r}$ and $5 \mathrm{t}$ had $66 \%$ protection, whereas compounds $5 \mathrm{a}, \mathbf{5 g}, \mathbf{5 h}, \mathbf{5 i}, 5 \mathrm{~m}$, $5 \mathbf{n}, \mathbf{5 p}, \mathbf{5 q}$ and $5 \mathrm{~s}$ were $50 \%$ protective in the anti-MES screen. In the neurotoxicity screen, compounds with $83-100 \%$ protection were selected and checked for neurotoxicity at the dose of $30 \mathrm{mg} \mathrm{kg}^{-1}$. None of the compounds displayed neurotoxicity, since they successfully passed the rotarod test without any sign of motor impairment (Table III). Bioevaluation led to correlation of the anticonvulsant screening with the basic structure of the compounds. In general, the disubstituted benzothiazole ring with 5,6-Cl substituents had higher potency than the 5-Cl and 6-F substitution. Disubstitution with $\mathrm{Cl}$ at the 5,6 position of the benzothiazole ring and $4-\mathrm{Br}, 3-\mathrm{Cl}$ substituents at the distant phenyl ring resulted in highly potent compounds, whereas replacement with $5-\mathrm{Cl}, 6-\mathrm{F}$ at the benzothiazole ring and $\mathrm{H}, 3-\mathrm{Cl}, 3-\mathrm{CH}_{3}, 2-\mathrm{OCH}_{3}, 3-\mathrm{OCH}_{3}, 4-\mathrm{CH}_{3}, 4-\mathrm{Cl}, 4-\mathrm{F}$ substituents at the 
Table II. Spectral characterization of synthesized compounds

\begin{tabular}{|c|c|c|c|}
\hline $\begin{array}{l}\text { Compd. } \\
\text { No. }\end{array}$ & FT-IR $\left(\mathrm{KBr}, \mathrm{cm}^{-1}\right)$ & ${ }^{1} \mathrm{H}$ NMR $\left(\mathrm{DMSO}-\mathrm{d}_{6}, \delta \mathrm{ppm}\right)$ & $\begin{array}{c}{ }^{13} \mathrm{C} \mathrm{NMR}\left(\mathrm{CDCl}_{3}, \mathrm{TMS},\right. \\
\delta \mathrm{ppm})\end{array}$ \\
\hline $5 a$ & $\begin{array}{l}3304 \text { (NH str.), } 3066 \\
(\mathrm{Ar}-\mathrm{CH}), 1577 \\
(\mathrm{C}=\mathrm{N}), 809(\mathrm{C}-\mathrm{Cl}) \\
657(\mathrm{C}-\mathrm{S}-\mathrm{C})\end{array}$ & $\begin{array}{l}6.96-7.59(\mathrm{~m}, 7 \mathrm{H}, \text { Ar- } \mathrm{H}), 9.18(\mathrm{~s}, \\
1 \mathrm{H}, \mathrm{NH}, \mathrm{D}_{2} \mathrm{O} \text { exchangeable), } 9.32 \\
\text { (bs, } 1 \mathrm{H}, \mathrm{NH}, \mathrm{D}_{2} \mathrm{O} \text { exchangeable) }\end{array}$ & $\begin{array}{l}185.63,164.70,156.68 \\
150.43,136.23,133.54 \\
130.75,125.78,123.89 \\
121.56,118.34\end{array}$ \\
\hline $5 b$ & $\begin{array}{l}3415 \text { (NH str.), } 3003 \\
(\mathrm{Ar}-\mathrm{CH}), 1545 \\
(\mathrm{C}=\mathrm{N}), 823(\mathrm{C}-\mathrm{Cl}) \\
617(\mathrm{C}-\mathrm{S}-\mathrm{C})\end{array}$ & $\begin{array}{l}6.87-7.64(\mathrm{~m}, 6 \mathrm{H}, \text { Ar- } \mathrm{H}), 9.23(\mathrm{~s}, \\
1 \mathrm{H}, \mathrm{NH}, \mathrm{D}_{2} \mathrm{O} \text { exchangeable), } 9.51 \\
\text { (bs, } 1 \mathrm{H}, \mathrm{NH}, \mathrm{D}_{2} \mathrm{O} \text { exchangeable) }\end{array}$ & $\begin{array}{l}184.78,164.54,154.65 \\
151.85,137.52,133.08 \\
130.31,128.45,124.65 \\
121.21,118.54,112.85\end{array}$ \\
\hline $5 c$ & $\begin{array}{l}3334 \text { (NH str.), } 3026 \\
(\text { Ar-CH), } 1551 \\
(\mathrm{C}=\mathrm{N}), 904(\mathrm{C}-\mathrm{Cl}) \\
643(\mathrm{C}-\mathrm{S}-\mathrm{C})\end{array}$ & $\begin{array}{l}6.91-7.79(\mathrm{~m}, 6 \mathrm{H}, \text { Ar- } \mathrm{H}), 9.19(\mathrm{~s}, \\
1 \mathrm{H}, \mathrm{NH}, \mathrm{D}_{2} \mathrm{O} \text { exchangeable), } 9.39 \\
\text { (bs, } 1 \mathrm{H}, \mathrm{NH}, \mathrm{D}_{2} \mathrm{O} \text { exchangeable) }\end{array}$ & $\begin{array}{l}182.24,166.47,154.79 \\
151.65,138.47,132.48 \\
130.86,126.55,123.23 \\
119.32\end{array}$ \\
\hline $5 d$ & $\begin{array}{l}3357 \text { (NH str.), } 3015 \\
(\text { Ar-CH), } 1564 \\
(\mathrm{C}=\mathrm{N}), 879(\mathrm{C}-\mathrm{Cl}) \\
634(\mathrm{C}-\mathrm{S}-\mathrm{C})\end{array}$ & $\begin{array}{l}6.92-7.71(\mathrm{~m}, 6 \mathrm{H}, \text { Ar- } \mathrm{H}), 9.16(\mathrm{~s}, \\
1 \mathrm{H}, \mathrm{NH}, \mathrm{D}_{2} \mathrm{O} \text { exchangeable), } 9.47 \\
\text { (bs, } 1 \mathrm{H}, \mathrm{NH}, \mathrm{D}_{2} \mathrm{O} \text { exchangeable) }\end{array}$ & $\begin{array}{l}186.65,164.88,154.84 \\
152.67,134.52,129.29 \\
126.24,123.70,117.85\end{array}$ \\
\hline $5 e$ & $\begin{array}{l}3319 \text { (NH str. }), 3034 \\
(\text { Ar-CH), } 1554 \\
(\mathrm{C}=\mathrm{N}), 1361(\mathrm{C}-\mathrm{F}) \\
865(\mathrm{C}-\mathrm{Cl}), 621 \\
(\mathrm{C}-\mathrm{S}-\mathrm{C})\end{array}$ & $\begin{array}{l}6.81-7.59(\mathrm{~m}, 6 \mathrm{H}, \text { Ar- } \mathrm{H}), 9.16(\mathrm{~s}, \\
1 \mathrm{H}, \mathrm{NH}, \mathrm{D}_{2} \mathrm{O} \text { exchangeable), } 9.45 \\
\text { (bs, } 1 \mathrm{H}, \mathrm{NH}, \mathrm{D}_{2} \mathrm{O} \text { exchangeable) }\end{array}$ & $\begin{array}{l}185.55,164.22,154.75 \\
151.63,140.66,136.54 \\
132.32,131.65,126.88 \\
123.74,121.55,116.84 \\
111.91\end{array}$ \\
\hline $5 f$ & $\begin{array}{l}3339 \text { (NH str.), } 3115 \\
(\text { Ar-CH), } 1561 \\
(\mathrm{C}=\mathrm{N}), 837(\mathrm{C}-\mathrm{Cl}) \\
637(\mathrm{C}-\mathrm{S}-\mathrm{C})\end{array}$ & $\begin{array}{l}3.11\left(\mathrm{~s}, 3 \mathrm{H}, \mathrm{CH}_{3}\right), 6.96-7.79(\mathrm{~m}, \\
6 \mathrm{H}, \mathrm{Ar}-\mathrm{H}), 9.09\left(\mathrm{~s}, 1 \mathrm{H}, \mathrm{NH}, \mathrm{D}_{2} \mathrm{O}\right. \\
\text { exchangeable), } 9.37(\mathrm{bs}, 1 \mathrm{H}, \mathrm{NH}, \\
\mathrm{D}_{2} \mathrm{O} \text { exchangeable) }\end{array}$ & $\begin{array}{l}184.54,164.74,154.70 \\
151.56,129.54,126.54 \\
123.65,121.54,118.21 \\
113.84,20.54\end{array}$ \\
\hline $5 \mathrm{~g}$ & $\begin{array}{l}3416 \text { (NH str.), } 3057 \\
(\text { Ar-CH), } 1504 \\
(\mathrm{C}=\mathrm{N}), 869(\mathrm{C}-\mathrm{Cl}) \\
659(\mathrm{C}-\mathrm{S}-\mathrm{C})\end{array}$ & $\begin{array}{l}3.19\left(\mathrm{~s}, 3 \mathrm{H}, \mathrm{CH}_{3}\right), 6.87-7.71(\mathrm{~m}, \\
6 \mathrm{H}, \text { Ar- } \mathrm{H}), 9.12\left(\mathrm{~s}, 1 \mathrm{H}, \mathrm{NH}, \mathrm{D}_{2} \mathrm{O}\right. \\
\text { exchangeable), } 9.41 \text { (bs, } 1 \mathrm{H}, \mathrm{NH}, \\
\mathrm{D}_{2} \mathrm{O} \text { exchangeable) }\end{array}$ & $\begin{array}{l}183.52,164.85,154.21 \\
151.65,130.54,128.33 \\
124.44,117.36,20.65\end{array}$ \\
\hline $5 \mathrm{~h}$ & $\begin{array}{l}3345 \text { (NH str.), } 3016 \\
\text { (Ar-CH), } 2933 \\
\text { (CH-aliph.), } 1528 \\
(\mathrm{C}=\mathrm{N}), 892(\mathrm{C}-\mathrm{Cl}) \\
687 \text { (C-S-C) }\end{array}$ & $\begin{array}{l}4.25\left(\mathrm{~s}, 3 \mathrm{H}, \mathrm{OCH}_{3}\right), 6.90-7.69(\mathrm{~m}, \\
6 \mathrm{H}, \mathrm{Ar}-\mathrm{H}), 9.32\left(\mathrm{~s}, 1 \mathrm{H}, \mathrm{NH}, \mathrm{D}_{2} \mathrm{O}\right. \\
\text { exchangeable), } 9.56(\mathrm{bs}, 1 \mathrm{H}, \mathrm{NH}, \\
\mathrm{D}_{2} \mathrm{O} \text { exchangeable) }\end{array}$ & $\begin{array}{l}\text { 182.22, 166.32, 155.74, } \\
151.69,130.54,129.54 \\
126.21,124.99,122.55 \\
120.68,116.44,114.54 \\
57.41\end{array}$ \\
\hline $5 \mathbf{i}$ & $\begin{array}{l}3315 \text { (NH str.), } 3009 \\
\text { (Ar-CH), } 2945 \\
\text { (CH-aliph.), } 1556 \\
(\mathrm{C}=\mathrm{N}), 837(\mathrm{C}-\mathrm{Cl}) \\
669 \text { (C-S-C) }\end{array}$ & $\begin{array}{l}4.16\left(\mathrm{~s}, 3 \mathrm{H}, \mathrm{OCH}_{3}\right), 6.78-7.54(\mathrm{~m}, \\
6 \mathrm{H}, \mathrm{Ar}-\mathrm{H}), 9.16\left(\mathrm{~s}, 1 \mathrm{H}, \mathrm{NH}, \mathrm{D}_{2} \mathrm{O}\right. \\
\text { exchangeable }), 9.43(\mathrm{bs}, 1 \mathrm{H}, \mathrm{NH}, \\
\mathrm{D}_{2} \mathrm{O} \text { exchangeable) }\end{array}$ & $\begin{array}{l}189.65,166.52,159.55 \\
154.79,140.22,133.85 \\
127.12,124.11,109.85 \\
104.32,54.23\end{array}$ \\
\hline $5 \mathbf{j}$ & $\begin{array}{l}3308 \text { (NH str.), } 3054 \\
\text { (Ar-CH), } 3004 \\
\text { (CH-aliph.), } 1579 \\
(\mathrm{C}=\mathrm{N}), 845(\mathrm{C}-\mathrm{Cl}) \\
698 \text { (C-S-C) }\end{array}$ & $\begin{array}{l}4.34\left(\mathrm{~s}, 3 \mathrm{H}, \mathrm{OCH}_{3}\right), 6.84-7.79(\mathrm{~m}, \\
6 \mathrm{H}, \mathrm{Ar}-\mathrm{H}), 9.10\left(\mathrm{~s}, 1 \mathrm{H}, \mathrm{NH}, \mathrm{D}_{2} \mathrm{O}\right. \\
\text { exchangeable }), 9.41(\mathrm{bs}, 1 \mathrm{H}, \mathrm{NH}, \\
\mathrm{D}_{2} \mathrm{O} \text { exchangeable) }\end{array}$ & $\begin{array}{l}\text { 181.22, 164.54, 156.12, } \\
154.85,152.65,131.55 \\
130.39,124.54,121.69 \\
118.45,113.54,110.69 \\
56.21\end{array}$ \\
\hline
\end{tabular}


3356 (NH str.), 3116

(Ar-CH), 2958

5k

(CH-aliph.), 1456

$(\mathrm{C}=\mathrm{N}), 1357$ (C-F), 684 (C-S-C)

3343 (NH str.), 3048

(Ar-CH), 1474

$51 \quad(\mathrm{C}=\mathrm{N}), 1369(\mathrm{C}-\mathrm{F})$, 656 (C-S-C), 563 (C-Br)

3323 (NH str.), 3007

(Ar-CH), 1465

$5 \mathrm{~m} \quad(\mathrm{C}=\mathrm{N}), 1342(\mathrm{C}-\mathrm{F})$, 852 (C-Cl), 647 (C-S-C)

3416 (NH str.), 3084

(Ar-CH), 1478

5n $(\mathrm{C}=\mathrm{N}), 1356(\mathrm{C}-\mathrm{F})$,

835 (C-Cl), 667

(C-S-C)

3423 (NH str.), 3091

(Ar-CH), 1504

$(\mathrm{C}=\mathrm{N}), 1345$ (C-F), 678 (C-S-C)

3478 (NH str.),

3167 (Ar-CH), 2967

5p (CH-aliph.), 1545

$(\mathrm{C}=\mathrm{N}), 1378$ (C-F),

667 (C-S-C)

3445 (NH str.), 3145

(Ar-CH), 2956

$5 q \quad$ (CH-aliph.), 1523

$(\mathrm{C}=\mathrm{N}), 1402$ (C-F), 679 (C-S-C)

3312 (NH str.),

3006 (Ar-CH), 2919

5r (CH-aliph.), 1535

$(\mathrm{C}=\mathrm{N}), 1392$ (C-F),

697 (C-S-C)

3359 (NH str.),

3019 (Ar-CH), 2958

$5 s$

(CH-aliph.), 1504

$(\mathrm{C}=\mathrm{N}), 1378$ (C-F), 684 (C-S-C)

3308 (NH str.), 3008 (Ar-CH), 2916

5t (CH-aliph.), 1515 $(\mathrm{C}=\mathrm{N}), 1354(\mathrm{C}-\mathrm{F})$, 673 (C-S-C)
6.67-7.74 (m, 7H, Ar-H), 9.24 (s, $1 \mathrm{H}, \mathrm{NH}, \mathrm{D}_{2} \mathrm{O}$ exchangeable), 9.58 (bs, $1 \mathrm{H}, \mathrm{NH}, \mathrm{D}_{2} \mathrm{O}$ exchangeable)

6.54-7.69 (m, 6H, Ar-H), 9.16 (s, $1 \mathrm{H}, \mathrm{NH}, \mathrm{D}_{2} \mathrm{O}$ exchangeable), 9.49 (bs, $1 \mathrm{H}, \mathrm{NH}, \mathrm{D}_{2} \mathrm{O}$ exchangeable)

6.48-7.63 (m, 6H, Ar-H), 9.23 (s, $1 \mathrm{H}, \mathrm{NH}, \mathrm{D}_{2} \mathrm{O}$ exchangeable), 9.58 (bs, $1 \mathrm{H}, \mathrm{NH}, \mathrm{D}_{2} \mathrm{O}$ exchangeable)

6.51-7.79 (m, 6H, Ar-H), 9.23 (s, $1 \mathrm{H}, \mathrm{NH}, \mathrm{D}_{2} \mathrm{O}$ exchangeable), 9.45 (bs, $1 \mathrm{H}, \mathrm{NH}, \mathrm{D}_{2} \mathrm{O}$ exchangeable)

6.79-7.85 (m, 6H, Ar-H), 9.18 (s, $1 \mathrm{H}, \mathrm{NH}, \mathrm{D}_{2} \mathrm{O}$ exchangeable), 9.59 (bs, $1 \mathrm{H}, \mathrm{NH}, \mathrm{D}_{2} \mathrm{O}$ exchangeable)

$3.21\left(\mathrm{~s}, 3 \mathrm{H}, \mathrm{CH}_{3}\right), 6.81-7.95(\mathrm{~m}$, $6 \mathrm{H}, \mathrm{Ar}-\mathrm{H}), 9.23$ (s, 1H, NH, $\mathrm{D}_{2} \mathrm{O}$ exchangeable), 9.46 (bs, $1 \mathrm{H}, \mathrm{NH}$, $\mathrm{D}_{2} \mathrm{O}$ exchangeable)

$3.24\left(\mathrm{~s}, 3 \mathrm{H}, \mathrm{CH}_{3}\right), 6.87-7.94(\mathrm{~m}$, $6 \mathrm{H}, \mathrm{Ar}-\mathrm{H}), 9.16$ (s, 1H, NH, D $\mathrm{O}$ exchangeable), 9.52 (bs, $1 \mathrm{H}, \mathrm{NH}$, $\mathrm{D}_{2} \mathrm{O}$ exchangeable)

$4.21\left(\mathrm{~s}, 3 \mathrm{H}, \mathrm{OCH}_{3}\right), 6.78-7.45(\mathrm{~m}$, $6 \mathrm{H}, \mathrm{Ar}-\mathrm{H}), 9.17$ (s, 1H, NH, $\mathrm{D}_{2} \mathrm{O}$ exchangeable), 9.38 (bs, $1 \mathrm{H}, \mathrm{NH}$, $\mathrm{D}_{2} \mathrm{O}$ exchangeable)

$4.24\left(\mathrm{~s}, 3 \mathrm{H}, \mathrm{OCH}_{3}\right), 6.72-7.49(\mathrm{~m}$, $6 \mathrm{H}, \mathrm{Ar}-\mathrm{H}), 9.25$ (s, 1H, NH, $\mathrm{D}_{2} \mathrm{O}$ exchangeable), 9.46 (bs, $1 \mathrm{H}, \mathrm{NH}$, $\mathrm{D}_{2} \mathrm{O}$ exchangeable)

$4.25\left(\mathrm{~s}, 3 \mathrm{H}, \mathrm{OCH}_{3}\right), 6.69-7.51(\mathrm{~m}$, $6 \mathrm{H}, \mathrm{Ar}-\mathrm{H}), 9.19$ (s, 1H, NH, D $\mathrm{O}$ exchangeable), 9.51 (bs, $1 \mathrm{H}, \mathrm{NH}$, $\mathrm{D}_{2} \mathrm{O}$ exchangeable)
184.65, 164.32, 154.69, 151.01, 149.64, 137.25, $129.81,124.24,122.64$, $116.69,112.14,110.54$

183.24, 165.35, 154.87, $151.54,147.56,136.56$, $133.58,130.56,128.21$, $124.54,121.45,116.24$, $112.54,110.94,108.37$ 184.62, 166.71, 154.75, 152.62, 149.59, 138.75, $133.94,131.27,126.35$, $122.45,120.57,118.41$, $116.28,112.70$

186.36, 166.25, 154.74, 153.67, 149.45, 136.12, $130.56,126.56,124.69$, $119.43,112.45,111.63$

186.24, 164.22, 153.63, 152.28, 150.27, 147.27, $142.69,139.57,135.16$, 126.77, 124.20, 122.65, $120.54,116.27,111.49$

183.54, 166.56, 154.21, 141.27, 129.57, 124.74, $120.74,114.75$, 112.74, 20.96

185.96, 166.34, 154.27, $150.65,138.96,130.24$, $125.29,117.41,112.63$, 21.64

188.24, 166.34, 155.67, $150.91,147.45,132.79$, $127.94,125.17,124.91$, 122.92, 120.35, 118.54, $114.14,111.79,56.34$

185.25, 166.34, 160.97, 155.39, 154.31, 150.63, $146.11,139.33,134.96$, 125.42, 117.52, 109.64, $105.34,55.74$

189.24, 166.27, 157.94, 154.87, 150.61, 134.61, 132.94, 124.67, 121.49, 118.37, 115.41, 111.27, 55.67 
N. Siddiqui et al.: Synthesis and preliminary screening of benzothiazol-2-yl thiadiazole derivatives for anticonvulsant activity, Acta Pharm. 59 (2009) 441-451.

distant phenyl ring led to a $50 \%$ decrease in potency. Disubstitution with 5,6- $\mathrm{Cl}$ and 5-Cl, 6-F at the benzothiazole ring and 4-F, $3-\mathrm{CH}_{3}$ at the distant phenyl ring resulted in compounds with highly significant activity. Compounds with disubstitution such as 5,6-Cl and $5-\mathrm{F}, 6-\mathrm{Cl}$ and $4-\mathrm{Br}, 4-\mathrm{Cl}$ or $4-\mathrm{OCH}_{3}, 2-\mathrm{OCH}_{3}$ at the distant phenyl ring had significant activity.

Enzyme estimation and histopathological studies of the selected compounds $5 \mathbf{b}$ and 5 c showing $100 \%$ protection were also performed to check the magnitude of liver toxicity and the data are presented in Table IV. The concentrations of alkaline phosphatase,

Table III. Anticonvulsant and neurotoxicity screening of compounds 5a-t

\begin{tabular}{|c|c|c|c|c|}
\hline \multirow{4}{*}{$\begin{array}{c}\text { Compd. } \\
\text { No. }\end{array}$} & \multicolumn{4}{|c|}{ Intraperitoneal injection in mice ${ }^{\mathrm{a}}$} \\
\hline & \multicolumn{2}{|c|}{ MES screen protection (\%) } & \multicolumn{2}{|c|}{ Neurotoxicity screen } \\
\hline & \multicolumn{4}{|c|}{ Time (h) } \\
\hline & 0.5 & 4 & 0.5 & 4 \\
\hline Control & - & - & - & - \\
\hline $5 a$ & 50 & 50 & NT & NT \\
\hline $5 b$ & 100 & 100 & - & - \\
\hline $5 c$ & 100 & 100 & - & - \\
\hline $5 d$ & 66 & 66 & NT & NT \\
\hline $5 e$ & 83 & 83 & - & - \\
\hline $5 f$ & 83 & 83 & - & - \\
\hline $5 \mathrm{~g}$ & 50 & 50 & NT & NT \\
\hline $5 \mathrm{~h}$ & 50 & 50 & NT & NT \\
\hline $5 i$ & 50 & 50 & NT & NT \\
\hline $5 j$ & 66 & 66 & NT & NT \\
\hline $5 k$ & 83 & 83 & - & - \\
\hline 51 & 66 & 66 & NT & NT \\
\hline $5 \mathrm{~m}$ & 50 & 50 & NT & NT \\
\hline $5 n$ & 50 & 50 & NT & NT \\
\hline 50 & 83 & 83 & - & - \\
\hline $5 p$ & 50 & 50 & NT & NT \\
\hline $5 q$ & 50 & 50 & NT & NT \\
\hline $5 r$ & 66 & 66 & NT & NT \\
\hline $5 s$ & 50 & 50 & NT & NT \\
\hline $5 t$ & 66 & 66 & NT & NT \\
\hline Phenytoin ${ }^{a}$ & 100 & 100 & - & - \\
\hline
\end{tabular}

Number of animals tested $(n=6)$.

a Dose of $30 \mathrm{mg} \mathrm{kg}^{-1}$ was administered i.p. The test compounds and standard drug were suspended in $1 \%$ Tween 80 or in $0.5 \%$ methylcellulose-water mixture. The animals were examined 0.5 and $4 \mathrm{~h}$ after administration. The (-) indicates the absence of activity and NT denotes not tested. 
N. Siddiqui et al.: Synthesis and preliminary screening of benzothiazol-2-yl thiadiazole derivatives for anticonvulsant activity, Acta Pharm. 59 (2009) 441-451.

Table IV. Liver toxicity studies of selected compounds ${ }^{a}$

\begin{tabular}{|c|c|c|c|c|}
\hline Treatment & $\begin{array}{l}\text { Alkaline phosphatase } \\
\text { (units L-1) }\end{array}$ & $\begin{array}{c}\text { SGOT } \\
\text { (units L-1) }\end{array}$ & $\begin{array}{c}\text { SGPT } \\
\text { (units L }{ }^{-1} \text { ) }\end{array}$ & $\begin{array}{l}\text { Total protein } \\
\left(\mathrm{g} \mathrm{dL}^{-1}\right)\end{array}$ \\
\hline Control & $13.06 \pm 0.25$ & $148.67 \pm 1.50$ & $27.67 \pm 0.840$ & $1.80 \pm 0.01$ \\
\hline $5 b$ & $12.82 \pm 0.16$ & $124.33 \pm 1.94^{b}$ & $25.33 \pm 0.49$ & $2.85 \pm 0.03^{b}$ \\
\hline $5 c$ & $15.31 \pm 0.23^{c}$ & $108.50 \pm 0.72^{c}$ & $33.17 \pm 0.870^{c}$ & $2.36 \pm 0.04^{c}$ \\
\hline
\end{tabular}

a Mean \pm SEM values $(n=6)$.

Significantly different from control: ${ }^{b} p<0.05,{ }^{\mathrm{c}} p<0.01$.

SGOT, SGPT and total protein were determined and the values are represented as mean \pm SEM. Compound $5 \mathbf{b}$ showed a significant change in the SGOT and total protein level compared to the control $(p<0.05)$, whereas compound $5 \mathrm{c}$ was found to increase significantly the concentration of all the liver enzymes estimated as well as the total protein level compared to the control $(p<0.01)$.

\section{CONCLUSIONS}

Thiadiazole incorporated benzothiazole derivatives can be regarded as a new structural class of anticonvulsant agents. Some of the compounds displayed encouraging activities in the MES test with less neurotoxicity and minimal effect on the liver. These compounds have promising prospects of future applications.

Acknowledgments. - The authors thank the All India Institute of Medical Sciences (AIIMS), New Delhi, India, for the histopathological studies and to the University Grants Commission (UGC, Government of India) for providing financial assistance.

\section{REFERENCES}

1. D. Hirtz, D. J. Thurman, K. Gwinn-Hardy, M. Mohamed, A. R. Chaudhuri and R. Zalutsky, How common are the »common« neurologic disorders?, Neurology 68 (2007) 326-337; DOI:10.1212/ 01.wnl.0000252807.38124.a3.

2. G. D. Cascino, Epilepsy: contemporary perspectives on evaluation and treatment, Mayo Clinic. Proc. 69 (1994) 1199-1211.

3. N. Siddiqui, S. N. Pandeya, S. A. Khan, J. P. Stables, A. Rana, M. Alam, M. F. Arshad and M. A. Bhat, Synthesis and anticonvulsant activity of sulfonamide derivatives-hydrophobic domain, Bioorg. Med. Chem. Lett. 17 (2007) 255-259; DOI:10.1016/j.bmcl.2006.09.053.

4. N. Siddiqui, A. Rana, S. A. Khan, M. A. Bhat and S. E. Haque, Synthesis of benzothiazole semicarbazones as novel anticonvulsants - The role of hydrophobic domain, Bioorg. Med. Chem. Lett. 17 (2007) 4178-4182; DOI:10.1016/j.bmcl.2007.05.048.

5. A. Rana, N. Siddiqui, S. A. Khan, S. E. Haque and M. A. Bhat, $N$-\{ $\{$ (6-substituted-1,3-benzothiazole-2-yl)amino]carbonothioyl\}-2/4-substituted benzamides: Synthesis and pharmacological evaluation, Eur. J. Med. Chem. 43 (2008) 1114-1122; DOI:10.1016/j.ejmech.2007.07.008. 
N. Siddiqui et al.: Synthesis and preliminary screening of benzothiazol-2-yl thiadiazole derivatives for anticonvulsant activity, Acta Pharm. 59 (2009) 441-451.

6. H. N. Dogan, A. Duran, S. Rollas, G. Sener, M. K. Uysal and D. Gülen, Synthesis of new 2,5-disubstituted-1,3,4-thiadiazoles and preliminary evaluation of anticonvulsant and antimicrobial activities, Bioorg. Med. Chem. 10 (2002) 2893-2898; DOI: 10.1016/S0968-0896(02)00143-8.

7. B. Masereel, S. Rolin, F. Abbate, A. Scozzafava and C. T. Supuran, Carbonic anhydrase inhibitors: Anticonvulsant sulfonamides incorporating valproyl and other lipophilic moieties, J. Med. Chem. 45 (2002) 312-320; DOI: 10.1021/jm0109199.

8. M. T. Silvina, C. M. Sung, E. B. Luis and L. E. Guillermina, Characterization of the anticonvulsant profile of valpromide derivatives, Bioorg. Med. Chem. 12 (2004) 3857-3869; DOI:10.1016/j. bmc.2004.05.003.

9. P. Yogeeswari, D. Sriram, V. Saraswat, R. J. Vaigunda, K. M. Mohan, S. Murugesan, R. Thirumurugan and J. P. Stables, Synthesis and anticonvulsant and neurotoxicity evaluation of $N^{4}$ -phthalimido phenyl (thio) semicarbazides, Eur. J. Pharm. Sci. 20 (2003) 341-346; DOI: 10.1016/ j.ejps. 2003.08.002.

10. Archana, V. K. Srivatava and K. Ashok, Synthesis of newer thiadiazolyl and thiazolidinonyl quinazolin-4(3H)-ones as potential anticonvulsant agents, Eur. J. Med. Chem. 37 (2002) 873-882; DOI:10.1016/S0223-5234(02)01389-2.

11. S. Reitman and S. A. Frankel, A colorimetric method for the determination of serum glutamic oxalacetic and glutamic pyruvic transaminases, Am. J. Clin. Pathol. 28 (1957) 56-63.

12. E. J. King and A. R. Armstrong, A convenient method for determining serum and bile phosphatase activity, Can. Med. Assoc. J. 31 (1934) 376-381.

13. D. S. Sheriff, Practical Clinical Biochemistry Methods and Interpretations, $1^{\text {st }}$ ed., Academa Publisher, Delhi 2004, pp. 38-39.

\section{$S A \check{Z} E T A K$}

\section{Sinteza i preliminarna ispitivanja antikonvulzivnog djelovanja derivata benzotiazol-2-il tiadiazola}

NADEEM SIDDIQUI, ARPANA RANA, SUROOR A. KHAN, S. EHTAISHAMUL HAQUE, M. FAIZ ARSHAD, SHARIQUE AHMED i WAQUAR AHSAN

U radu je opisano dizajniranje i sinteza različitih $N$-(5-klor-6-supstituiranih benzotiazol-2-il)-N'-(supstituiranih fenil)-[1,3,4]tiadiazol-2,5-diamina (5a-t) polazeći od odgovarajućih acetofenona. Strukture spojeva određene su na temelju spektroskopskih podataka i elementarne analize. Ispitano je antikonvulzivno djelovanje svih novosintetiziranih spojeva i uspoređeno s djelovanjem natrijeve soli fenitoina. Spojevi 5b i 5c pružaju potpunu zaštitu od konvulzija uzrokovanih MES-om, a svi spojevi štite od konvulzija u rasponu od 50 do $100 \%$.

Ključne riječi: benzotiazol, tiadiazol, antikonvulziv, neurotoksičnost, hepatotoksičnost

Department of Pharmaceutical Chemistry, Faculty of Pharmacy, Hamdard University, New Delhi-110062, India

Department of Pharmacology, Faculty of Pharmacy, Hamdard University, New Delhi-110062, India

Department of Biochemistry, Faculty of Medicine, $7^{\text {th }}$ October University, Misurata, Libya 